\title{
Emerging Technology Commission on Al report
}

\author{
Andrew D. Smith ${ }^{1} \cdot$ George Shih ${ }^{2}$
}

Accepted: 18 November 2020

(c) Springer Science+Business Media, LLC, part of Springer Nature 2021

\section{List of all active SAR ETC on AI members:}

\section{Andrew D. Smith MD PhD}

Co-Chair of SAR ETC on AI

Institution: University of Alabama at Birmingham

Position: Professor and Vice Chair of Clinical Research

Email: andrewdennissmith@uabmc.edu

\section{George Shih MD}

Co-Chair of SAR ETC on AI

Institution: Weill Cornell Medical College

Position: Associate Professor and VC of Informatics

Email: george@cornellradiology.org

3. Sarah Bastawrous DO

Institution: University of Washington

Position: Associate Professor

Email:ssheikh@uw.edu

\section{Paul Chang MD}

Institution: University of Chicago School of Medicine

Position: Professor and Vice Chair of Radiology Informatics

Email: pchang@ radiology.bsd.uchicago.edu

AI Interests:

\section{Marc Kohli MD}

Position: Associate Professor and Director of Informatics Institution: University of California San Francisco

Email:marc.kohli@ucsf.edu

\section{Andrew D. Smith}

andrewdennissmith@uabmc.edu

George Shih

george@cornellradiology.org

1 University of Alabama At Birmingham, Birmingham, AL, USA

2 Weill Cornell Medical College, New York, NY, USA
6. Mark Kovacs MD

Institution: Medical University of South Carolina

Position: Assistant Professor, IT Medical Director,

Radiology

Email: kovacsm@musc.edu

\section{Arun Krishnaraj MD}

Institution: University of Virginia

Position: Associate Professor, Vice Chair of Quality \&

Safety, Director Body Imaging

Email:ak8jj@hscmail.mcc.virginia.edu

8. Susanna Lee MD

Institution: Massachusetts General Hospital

Position: Associate Professor

Email: slee0@mgh.harvard.org

9. Thomas Loehfelm MD PhD

Institution: UC Davis Health

Position: Associate Professor

Email: twloehfelm@ucdavis.edu

10. John Mongan MD

Institution: UCSF

Position: Associate Professor and Associate Chair of

Translational Informatics

Email: john.mongan@ucsf.edu

11. Paul Murphy MD PhD

Institution: UCSD

Position: Assistant Professor

Email:pmmurphy@ucsd.edu

12. Doug Nachand MD

Institution: Cleveland Clinic

Position: Assistant Professor (Early Career)

Email: nachand@ccf.org

\section{Stacy O'Connor MD MPH MMSc}

Institution: Medical College of Wisconsin 
Position: Associate Professor and Medical Director of Radiology IT Operations

Email: soconnor@mcw.edu

14. Bhavik Patel MD MBA

Institution: Mayo Clinic in Arizona

Position: Senior Associate Consultant

Email: patel.bhavik@mayo.edu

15. Iva Petkovska MD

Institution: MSKCC

Position: Assistant Professor

Email: petkovsi@mskcc.org

16. Perry J. Pickhardt MD

Institution: University of Wisconsin

Position: Professor and Medical Director of Oncologic Imaging

Email: ppickhardt2@uwhealth.org

\section{Andrea Rockall MBBS}

Institution: Imperial College of London

Position: Professor and Clinical Chair of Radiology

Email: a.rockall@imperial.ac.uk

18. Daniel Rubin MD MS

Institution: Stanford University

Position: Professor of Biomedical Data Science, Radiol-

ogy, and Medicine

Email: dlrubin@stanford.edu

19. Ronald M. Summers MD PhD

Institution: National Institutes of Health Clinical Center

Position: Senior Investigator

Email:rms@nih.gov

\section{Hanna M. Zafar MD MHS}

Institution: University of Pennsylvania

Position: Associate Professor and Associate Vice Chair Quality

Email: hanna.zafar@uphs.upenn.edu

\section{Marc Zins MD}

Institution: Groupe Hospitalier Paris Saint Joseph

Position: Professor and Chairman

Email: mzins@hpsj.fr

\section{Look back}

The Emerging Technology Commission (ETC) was created by the Society of Abdominal Radiology (SAR) as a mechanism to improve patient care, education, and research in new or evolving technologies that impact abdominal radiology. The first ETC was focused on artificial intelligence (AI) and was proposed in 2019. The primary goals of the SAR ETC on AI are to validate, develop, and educate on $\mathrm{AI}$, machine learning, and deep learning technologies that will impact the clinical practice of abdominal radiologists and the quality of patient care they deliver. A diverse group of abdominal imaging faculty with experience in AI was assembled to take on this challenge.

In anticipation of the 2020 SAR Annual Meeting, the ETC on AI proposed the SAR AI Challenge, a competition and collaboration with the American College of Radiology (ACR) Data Science Institute (DSI). Prior to the annual meeting, members proposed use cases or clinical scenarios in abdominal radiology where AI tools should be developed. Over 50 use cases from 30 institutions and 4 countries were submitted and reviewed by relevant disease-focused panels (DFPs) and the ETC on AI. The top three abdominal use cases were presented at the "SAR Tank" event at the 2020 Annual Meeting, and the winners were selected by a panel of judges.

The SAR ETC on AI created a homepage on the SAR website that includes the SAR Tank competition from 2020, a photo of the winners, and multiple video presentations from those receiving honorable mention [1]. The SAR ETC on AI had a series of plenary lectures and workshops at the 2020 Annual Meeting and held an AI Master Class where participants had hands-on experience running a variety of AI algorithms.

Members of the SAR ETC on AI conducted a multi-institutional study entitled "Comparative effectiveness of advanced cancer longitudinal response evaluation methods: artificial intelligence-assisted vs. standard-of-care." The project included 28 radiologists and 20 oncologic providers from 21 institutions and won the Best New Frontiers Scientific Presentation Award at the 2020 SAR Annual Meeting.

Initial challenges for the SAR ETC on AI were in identifying appropriate projects to pursue, but that hurdle was crossed by superb ideas from members. There have been challenges on how to handle conflicts of interest from members and challenges with finding a fair and equitable method to incorporate industry consultants in the SAR ETC on AI. Other challenges include lack of funding for most research projects, decreased activity during the COVID-19 pandemic, and difficulties in sharing and gathering multi-institutional data sets, annotating or preparing data sets for AI algorithm training, and information technology hurdles for distributed sharing of AI algorithms or federated learning.

\section{Look ahead}

The SAR ETC on AI plans to provide another series of plenary lectures and workshops on AI topics and will offer an additional hands-on workshop. Team members are also 
working on educational manuscripts including a strengths, weaknesses, opportunities and threats (SWOT) analysis of $\mathrm{AI}$ in abdominal radiology. Other team members are working educational activities for medical students and residents interested in AI and abdominal radiology.

The SAR ETC on AI has several collaborative projects planned to include the following:

1. Develop and validate a multi-institutional distributed AI sharing model for automated body composition metrics.

2. Establish a partnership between the SAR and the Radiological Society of North America (RSNA) for an AI challenge focused on a topic in abdominal imaging.

3. Develop and validate an open-source AI algorithm for CT image series identification using a multi-institutional data set of single energy and dual/spectral CT abdominal images.

4. Validate the use of deep learning reconstruction to reduce noise and improve detection of cancer-related image findings on a multi-institutional data set of dual energy/spectral CT images. The emphasis will be on the low energy images that have high contrast and high noise.
5. Explore AI algorithm development for prostate MRI in combination with the SAR DFP on prostate cancer using the award winning use case from the SAR Tank as a point of reference [1].

The early success and challenges faced by the SAR ETC on AI are expected to lead to future work that will positively impact the clinical practice of abdominal radiologists and the quality of patient care they deliver.

\section{Reference}

1. Society of Abdominal Radiology Emerging Technology Commission on Artificial Intelligence (2021, January 21). Retrieved from https://abdominalradiology.org/sar-subpages/dfp-panels/.

Publisher's Note Springer Nature remains neutral with regard to jurisdictional claims in published maps and institutional affiliations. 